\title{
Erratum: Cosmological models in energy-momentum-squared gravity [Phys. Rev. D 96, 123517 (2017)]
}

\author{
Charles V. R. Board and John D. Barrow
}

(Received 7 December 2018; published 28 December 2018)

DOI: $10.1103 /$ PhysRevD.98.129902

The right-hand side of Eq. (24) is corrected by inserting a missing factor of $\left(1+3 w^{2}\right)^{n-1}$ into the second term of the numerator and a missing factor of $n$ into the second term of the denominator. Corrected factors are inserted into Eqs. (25)-(28) and (30)-(32) as a consequence. The discussion and conclusions remain unchanged.

Equation (24) should read

$$
\dot{\rho}=-3 \frac{\dot{a}}{a} \rho(1+w)\left[\frac{\kappa+\eta \rho^{2 n-1} n(1+3 w)\left(1+3 w^{2}\right)^{n-1}}{\kappa+2 \eta \rho^{2 n-1} n A(n, w)}\right] .
$$

Equation (25) should read

$$
\dot{\rho}\left(\frac{1}{\rho}+\frac{2 \eta n A\left(n, \frac{-1}{3}\right)}{\kappa} \rho^{2 n-2}\right)=-2 \frac{\dot{a}}{a}
$$

Equation (26) should read

$$
\frac{d}{d t}\left(\ln (\rho)-\frac{\eta n\left(\frac{4}{3}\right)^{n}}{(2 n-1) \kappa} \rho^{2 n-1}\right)=\frac{d}{d t}\left(\ln \left(a^{-2}\right)\right)
$$

Equation (27) should read

$$
\rho \exp \left(-\frac{\eta n\left(\frac{4}{3}\right)^{n}}{(2 n-1) \kappa} \rho^{2 n-1}\right)=C a^{-2}
$$

Equation (28) should read

$$
(1+3 w)\left(1+3 w^{2}\right)^{n-1}=2 A(n, w) .
$$

Equation (30) should read

$$
n(1+3 w)\left(1+3 w^{2}\right)^{n-1}=A(n, w) .
$$

Equation (31) should read

$$
\frac{d}{d t}\left(\ln \left(\kappa \rho+n \eta \rho^{2 n}\left(1+3 w_{*}\right)\left(1+3 w_{*}^{2}\right)^{n-1}\right)\right)=\frac{d}{d t}\left(\ln \left(a^{-3\left(1+w_{*}\right.}\right)\right) .
$$

Equation (32) should read

$$
\kappa \rho+n \eta \rho^{2 n}\left(1+3 w_{*}\right)\left(1+3 w_{*}^{2}\right)^{n-1}=C a^{-3\left(1+w_{*}\right)} .
$$

We thank Sebastian Bahamonde, Mihai Marciu, and Prabir Rudra for bringing the issue to our attention. 